\title{
Nonlinear anisotropy growth in Bianchi-I spacetime in metric $f(R)$ cosmology
}

\author{
Kaushik Bhattacharya, Saikat Chakraborty * \\ Department of Physics, Indian Institute of Technology, \\ Kanpur 208016, India
}

January 9, 2019

\begin{abstract}
The present work is related to anisotropic cosmological evolution in metric $f(R)$ theory of gravity. The initial part of the paper develops the general cosmological dynamics of homogeneous anisotropic BianchiI spacetime in $f(R)$ cosmology. The anisotropic spacetime is pervaded by a barotropic fluid which has isotropic pressure. The paper predicts nonlinear growth of anisotropy in such spacetimes. In the later part of the paper we display the predictive power of the nonlinear differential equation responsible for the cosmological anisotropy growth in various relevant cases. We present the exact solutions of anisotropy growth in Starobinsky inflation driven by quadratic gravity and exponential gravity theory. Semi-analytical results are presented for the contraction phase in quadratic gravity bounce. The various examples of anisotropy growth in Bianchi-I model universe shows the complex nature of the problem at hand.
\end{abstract}

\section{Introduction}

The issue related to stability of homogeneous and isotropic cosmological solutions with respect to small anisotropy has been studied intensely in theoretical cosmology [1], [2], [3], [4]. Behavior of small anisotropy has been studied in cosmological

${ }^{*}$ E-mail: kaushikb, snilch@iitk.ac.in 
models, using general relativity (GR), in the contexts of inflation [5], [6], [7], [8], [9] and pre-bounce ekpyrotic contraction phase [10], [11], [12], [13]. In the context of inflation the 'No-Hair' conjecture asserts that any pre-existing anisotropy must asymptotically die out in an inflating universe. Wald has been able to prove the conjecture for all the Bianchi models except Bianchi-IX [14] which requires a large cosmological constant to isotropize the spacetime. In a contracting universe, provided the universe is dominated by a matter component mimicking an ultra stiff barotropic fluid, growth of small anisotropy is suppressed with respect to that of the Hubble parameter. In absence of any such fluid in a contracting phase, small anisotropy grows large and dominates over all other matter components. This leads to the Belinsky-Khalatnikov-Lifshitz (BKL) instability [15], foiling the bounce. Mathematically, it can be shown that in presence of a slowly rolling scalar field the isotropic de-Sitter solution is an attractor for an expanding universe and in presence of a fast rolling scalar field the isotropic power law solution (for the scale-factor) is an attractor for contracting universe. Therefore an inflationary scenario is usually realized by a slowly rolling scalar field and an ekpyrotic scenario is usually realized by a fast rolling scalar field [16], 17], [18], [19], [20].

In the present work we have analyzed the evolution of spacetime anisotropy in $f(R)$ gravity 1 , where the analysis becomes significantly more involved than that of models based on GR. Some attempts in modified, quadratic gravity 22, 23 do discuss about anisotropic cosmologies while analyzing the past stages of a cosmological system near the singularity. In these works the authors show that near the singularity the universe may have an anisotropic mode of existence. The papers in general do not address a cosmological bounce scenario. Although previously there have been some progress in generalizing the no-hair theorem to incorporate higher order gravity theories [24], [25], [26], [27] and some applications of dynamical system analysis to understand anisotropic cosmology in higher order gravity 28], 29], 30], the previous attempts missed an important property of anisotropic cosmological dynamics related to nonlinear growth of anisotropy in the homogeneous and anisotropic Bianchi-I type of spacetime. In the present paper we first show analytically that in Starobinsky inflation any initial anisotropy will rapidly fade away.

It is then shown that in contraction phases in Bianchi-I metric where speci-

\footnotetext{
${ }^{1}$ For a general understanding of modified gravity theories one can look at the review in Ref. [21].
} 
fying an unique scale-factor for contraction does not always yield a unique cosmological development. This result is possible in $f(R)$ cosmology and in GR one cannot have this property. This non-uniqueness of cosmological development corresponding to a specific scale-factor opens up a new problem as cosmological evolution becomes more complex conceptually and as a consequence only simple $f(R)$ models can be semi-analytically solved. Any $f(R)$ model which is a higher order polynomial in $R$ compared to the quadratic $f(R)$ model requires a complete numerical solution for anisotropy growth. We show our result in quadratic $f(R)$ model, which is gravitationally unstable if it has to accommodate a cosmological bounce. Our work may be taken as an effective toy model which is used to crack a formidable problem in cosmological dynamics. To our understanding the above mentioned topics are discussed for the first time in full generality in the present paper.

We also present some preliminary results of non-linear anisotropy growth in exponential gravity models. The exponential gravity model has two exact solutions. One exact solution is a bouncing solution in presence of matter and the other exact solution is a expanding universe solution at a de-Sitter point. In these cases the anisotropy generation equation turns out to be a transcendental equation. We present some simple solutions in this case probing the nature of growth of small initial anisotropy.

The material in the paper is organized in the following way. The second section discusses about the basics of Bianchi-I spacetimes and sets the notations and conventions followed throughout the paper. In section 3 we present the general formalism of homogeneous and anisotropic cosmological dynamics in metric $f(R)$ cosmology. This part contains important results. In this section for the first time one comes across the complex nature of anisotropy development. In section 4 we present the results related to nonlinear anisotropy growth in quadratic $f(R)$ theory induced inflation and cosmological bounce. Section 5 presents the results of anisotropy growth for some exact results in exponential gravity. The next section is the concluding section where we summarize the results obtained in the paper. 


\section{The anisotropic Bianchi-I metric and its prop- erties}

For our analysis, we have used the metric for Bianchi-I spacetime,

$$
d s^{2}=-d t^{2}+a_{1}^{2}(t) d x_{1}^{2}+a_{2}^{2}(t) d x_{2}^{2}+a_{3}^{2}(t) d x_{3}^{2},
$$

where $a_{1}(t), a_{2}(t)$ and $a_{3}(t)$ are the different scale-factors, whose relative differences specify the amount of anisotropy in the evolving universe. Existence of such anisotropic cosmological models in higher order gravity theories have been extensively studied in literature [31], [32], [33], [34]. In most of the earlier attempts the authors have tried to find out the nature of anisotropic spacetimes using various forms of anisotropic metric and using various forms of gravitational Lagrangians. In the present paper we show that the previous attempts have missed a vital ingredient in anisotropic expansion/contraction. The effect we discuss is clearly visible in Bianchi-I spacetime, but we think similar effects may be present in other anisotropic cosmological models.

In this paper we will assume the presence of a perfect hydrodynamic fluid, in the Bianchi type-I spacetime, whose energy-momentum tensor (EMT)is given by $T_{\mu \nu}=(\rho+P) u_{\mu} u_{\nu}+P g_{\mu \nu}$, where $\rho$ is the energy-density and $P$ specifies isotropic pressure of the perfect fluid. The 4-velocity of the fluid element is given by $u_{\mu}$, which being a time-like vector is normalized as $u^{\mu} u_{\mu}=-1$. Although the spacetime metric is anisotropic the fluid which pervades the spacetime is assumed to be isotropic. In this paper we will assume a barotropic equation of state for the perfect fluid, $P=\omega \rho$, where $\omega$ specifies the barotropic ratio.

One can rewrite the form of the anisotropic metric, given in Eq. (1), in terms of the (geometric) average of the three scale-factors given by $a(t)=\left[a_{1}(t) a_{2}(t) a_{3}\right]^{1 / 3}$. The three different scale-factors in terms of the geometric average scale-factor can be written as, $a_{i}(t)=a(t) e^{\beta_{i}(t)}$, where $i=1,2,32$. The time dependent functions $\beta_{i}(t)$ specify the anisotropy in the metric and they are constrained as

$$
\beta_{1}+\beta_{2}+\beta_{3}=0
$$

Using the above relations one can now rewrite the metric given in Eq. (1) as

$$
d s^{2}=-d t^{2}+a^{2}(t)\left[e^{2 \beta_{1}(t)} d x_{1}^{2}+e^{2 \beta_{2}(t)} d x_{2}^{2}+e^{2 \beta_{3}(t)} d x_{3}^{2}\right] .
$$

\footnotetext{
${ }^{2}$ Latin alphabets as $i$ and $j$ run from 1 to 3 where as Greek alphabets run from 0 to 3 .
} 
In this notation one can define the Hubble parameter, as an arithmetic average, and its time-derivative as

$$
H(t) \equiv \frac{1}{3}\left(\frac{\dot{a}_{1}}{a_{1}}+\frac{\dot{a}_{2}}{a_{2}}+\frac{\dot{a}_{3}}{a_{3}}\right)=\frac{\dot{a}}{a}, \quad \dot{H}(t)=\frac{\ddot{a}}{a}-\frac{\dot{a}^{2}}{a^{2}} .
$$

Mainly for the sake of brevity, henceforth in this article we will omit the word average (either geometric or arithmetic) before scale-factor or Hubble parameter. In presence of anisotropy the Ricci scalar turns out to be

$$
R=6\left(\dot{H}+2 H^{2}\right)+\left(\dot{\beta}_{1}^{2}+\dot{\beta}_{2}{ }^{2}+\dot{\beta}_{3}{ }^{2}\right) .
$$

In the next section we formulate the anisotropic cosmological dynamics guided by metric $f(R)$ theory. The fact that the derivatives of the anisotropy parameters are themselves present in the expression of the Ricci scalar will make anisotropic cosmological dynamics much more involved in $f(R)$ gravity, compared to the general relativistic case.

\section{Formulation of anisotropic cosmological dy- namics guided by metric $f(R)$ theory}

The field equation in $f(R)$ gravity is

$$
G_{\nu}^{\mu}=\frac{\kappa}{f^{\prime}(R)}\left[T_{\nu}^{\mu}+T_{\nu(\text { curv })}^{\mu}\right]
$$

where $G_{\nu}^{\mu}$ is the Einstein tensor and $T_{\nu \text { (curv) }}^{\mu}$ is the energy momentum tensor due to curvature. Here $\kappa=8 \pi G$, where $G$ is the Newton's gravitational constant and is related to the Planck mass $M_{P}$ via $G=1 / M_{P}^{2}$. In the present paper we will approximately use $M_{P} \approx 10^{19} \mathrm{GeV}$. The prime on top right hand side of any function represents the ordinary derivative of that function with respect to the Ricci scalar $R$. In particular

$$
T_{\nu(\mathrm{curv})}^{\mu} \equiv \frac{1}{\kappa}\left[-\left(\frac{R f^{\prime}(R)-f(R)}{2}+\square f^{\prime}(R)\right) \delta_{\nu}^{\mu}+g^{\mu \alpha} D_{\alpha} D_{\nu} f^{\prime}(R)\right]
$$

where $D_{\mu} A_{\nu}$ is the covariant derivative of the covariant 4-vector $A_{\nu}$ and $\square \equiv$ $g^{\alpha \beta} D_{\alpha} D_{\beta}$. The $0-0$ component of the field equation in $f(R)$ theory in an anisotropic spacetime is then given as, $G_{0}^{0}=-\frac{\kappa}{f^{\prime}(R)}\left(\rho+\rho_{\text {curv }}\right)$, where

$$
\rho_{\text {curv }}=\frac{1}{\kappa}\left[\frac{R f^{\prime}(R)-f(R)}{2}-3 H \dot{R} f^{\prime \prime}(R)\right] \text {. }
$$


The other three equations, specifying the $i-j$ components become, $G^{i}{ }_{j}=$ $\frac{\kappa}{f^{\prime}(R)}\left(T_{j}^{i}+T_{j \text { (curv) }}^{i}\right)$, where $i, j=1,2,3$. Here $T_{j}^{i}=P \delta_{j}^{i}$ stands for pressure of the perfect hydrodynamic fluid(s) whose EMT(s) has(have) the same form as specified in section 2. The form of $T^{i}{ }_{j \text { (curv) }}$ is given as

$$
\begin{aligned}
\kappa T_{j(\text { curv })}^{i}= & -\left[\frac{R f^{\prime}(R)-f(R)}{2}-\ddot{R} f^{\prime \prime}(R)-\dot{R}^{2} f^{\prime \prime \prime}(R)-2 H \dot{R} f^{\prime \prime}(R)\right] \delta_{j}^{i} \\
& -B_{j}^{i} \dot{R} f^{\prime \prime}(R),
\end{aligned}
$$

where the components of the tensor $B_{j}^{i}$ are defined as

$$
B_{1}^{1}=\dot{\beta}_{1}, B_{2}^{2}=\dot{\beta}_{2}, B_{3}^{3}=\dot{\beta}_{3}, B_{j}^{i}=0, \text { if }, i \neq j
$$

In terms of the above quantities one can now write,

$$
G_{j}^{i}=\frac{\kappa}{f^{\prime}(R)}\left(P+P_{\text {curv }}\right) \delta_{j}^{i}-B_{j}^{i} \dot{R} f^{\prime \prime}(R),
$$

where

$$
P_{\text {curv }}=\frac{\dot{R}^{2} f^{\prime \prime \prime}+2 H \dot{R} f^{\prime \prime}+\ddot{R} f^{\prime \prime}}{\kappa}-\frac{R f^{\prime}-f}{2 \kappa} .
$$

In terms of the Hubble parameter and the anisotropy parameter, the $0-0$ component of the field equation becomes

$$
H^{2}=\frac{\kappa}{3 f^{\prime}(R)}\left(\rho+\rho_{\text {curv }}\right)+\frac{1}{6} \sum_{i=1}^{3} \dot{\beta}_{i}^{2},
$$

while Eq. 11] becomes

$$
2 \dot{H}+3 H^{2}-3 H \dot{\beta}_{i}-\ddot{\beta}_{i}+\frac{1}{2} \sum_{i=1}^{3} \dot{\beta}_{i}^{2}=-\frac{\kappa}{f^{\prime}(R)}\left(P+P_{\text {curv }}\right)+\dot{\beta}_{i} \dot{R} \frac{f^{\prime \prime}(R)}{f^{\prime}(R)} .
$$

Adding the three equations, corresponding to each value of the index $i$ in the above expression, one gets

$$
2 \dot{H}+3 H^{2}=-\frac{\kappa}{f^{\prime}(R)}\left(P+P_{\text {curv }}\right)-\frac{1}{2} \sum_{i=1}^{3} \dot{\beta}_{i}^{2} .
$$

If one uses Eq. 13 in the above equation then one gets $\dot{H}$ as

$$
\dot{H}=-\frac{\kappa}{2 f^{\prime}(R)}\left[(1+\omega) \rho+\left(\rho_{\text {curv }}+P_{\text {curv }}\right)\right]-\frac{1}{2} \sum_{i=1}^{3} \dot{\beta}_{i}^{2},
$$


where $P=\omega \rho$ has been used. In the present case we define the anisotropy factor $x$ as

$$
x^{2}(t) \equiv \sum_{i=1}^{3} \dot{\beta}_{i}^{2}(t) .
$$

For an isotropic universe $x^{2}=0$ for all values of $t$, implying that all the $\beta_{i}$ 's are constant in time. In such a case one can appropriately make (time-independent) coordinate rescaling in an appropriate way to make the spacetime look exactly like the Friedmann-Lemaitre-Robertson-Walker (FLRW) spacetime. Using Eq. (2) and Eq. (14) and the above definition of the anisotropy factor one can show that $x$ satisfies the differential equation:

$$
\dot{x}+\left(3 H+\frac{\dot{f}^{\prime}(R)}{f^{\prime}(R)}\right) x=0,
$$

whose (nontrivial) solution must be like

$$
x=\frac{b}{a^{3}(t) f^{\prime}(R)},
$$

where $b$ is a real integration constant. The above equation contains the most important theoretical ingredient of the present paper. The Ricci scalar in the present case can be written as $R=6\left(\dot{H}+2 H^{2}\right)+x^{2}$ which depends on $x$ and the last equation shows $x$ is a function of $R$ in $f(R)$ gravity. As a consequence of the above relation in $f(R)$ gravity, one cannot define an unique anisotropy dynamics. For any given $f(R)$ gravity, in general multiple time evolutions of the anisotropy factor $x$ is possible, each corresponding to a different equation of state for the barotropic fluid. In the present case the time derivative of the Ricci scalar is

$$
\dot{R}=\frac{6\left(\ddot{H}+4 H \dot{H}-H x^{2}\right)}{\left(1+2 \frac{f^{\prime \prime}(R)}{f^{\prime}(R)} x^{2}\right)} .
$$

Working out similarly one can write,

$$
\ddot{R}=\frac{6\left(\dddot{H}+4 H \ddot{H}+4 \dot{H}^{2}\right)-2\left[\left(3 \dot{H}+\frac{\dot{R}^{2} f^{\prime \prime \prime}}{f^{\prime}}-\frac{\dot{f}^{\prime 2}}{f^{\prime 2}}\right)-2\left(3 H+\frac{\dot{f}^{\prime}}{f^{\prime}}\right)^{2}\right] x^{2}}{\left(1+2 x^{2} \frac{f^{\prime \prime}}{f^{\prime}}\right)} .
$$

The above equations show that once we know the form of $x$ in terms of the scalefactor, we can write the values of $R, \dot{R}$ and $\ddot{R}$ in terms of the scale-factor. The 
cosmological dynamics of anisotropic $f(R)$ theory is encoded in Eq. (16), Eq. (19) and the energy-momentum conservation equation

$$
\dot{\rho}+3 H \rho(1+\omega)=0
$$

The specification of $\omega$ and this set of three equations and the initial conditions specifying $a, \dot{a}, \ddot{a}, \dddot{a}, b$ and initial $\rho$ are enough to specify the anisotropic dynamics in $f(R)$ cosmology if Eq. (19) has an unique root. If Eq. (19) does not have an unique root then the initial conditions must have to be enhanced. In the next section we will show when the above list of initial conditions require to be enhanced.

The first order differential equation in Eq. 18) specifies the growth of anisotropy in metric $f(R)$ gravity models where spacetime is specified by a Bianchi-I model. From the form of the equation it is seen that the amount of nonlinearity in Eq. (18) depends upon time. It can be noted that $x=0$ have some interesting properties. The first thing to note about this point is that at $x=0$ one always has $\dot{x}=0$. The other interesting properties about this point are as follows.

1. If the system resides at the point $x=0$ then is is impossible to perturb the system to have non-zero values of $x$. The system can have $x=0$ value only when $b=0$, and $b$ is specified by the initial condition. Consequently if the initial condition is such that $x=0$ then there will be no anisotropy growth in the future.

2. On the other hand if the initial condition is such that $b \neq 0$ then the system will never reach $x=0$ unless $a^{3}(t) f^{\prime}(R)$ diverges in finite time, signifying a cosmological singularity.

As $x$ cannot be zero in the future if $b \neq 0$ in a non-singular cosmology, the important parameter which keeps track of effective anisotropy is given by the factor $x^{2} / H^{2}$. From Eq. (13), Eq. (15) and the expression of the Ricci scalar, $R$, it can be verified that when $x^{2} / H^{2} \ll 1$ one can safely neglect the effect of anisotropy in the cosmological dynamics of Bianchi-I type models. 


\section{Evolution of the anisotropic factor $x(t)$ in quadratic gravity}

In this section we will focus on quadratic gravity

$$
f(R)=R+\alpha R^{2}
$$

where $\alpha$ is a real number. Although this is a simple form of $f(R)$ but it can be used to model cosmological inflation as well as cosmological bounce for positive and negative signs of the constant $\alpha$ respectively ${ }^{3}$. In this section we will determine the evolution of $x(t)$ in quadratic gravity. The technique of evolution of $x(t)$ in higher order gravity will be similar but much more involved. For higher order polynomial gravity the order of the algebraic equation yielding the roots of $x(t)$ may be five (or higher) and consequently there does not exist any general algebraic formalism yielding those roots.

From Eq. 19) one can easily verify that the algebraic equation specifying $x(t)$ in quadratic gravity is a cubic equation of the form:

$$
x^{3}+A_{1} x+A_{2}=0,
$$

where

$$
\begin{aligned}
& A_{1}=6\left(\dot{H}+2 H^{2}\right)+\frac{1}{2 \alpha}, \\
& A_{2}=-\frac{b}{2 \alpha a^{3}} .
\end{aligned}
$$

The discriminant, $\Delta$, specifying the roots and their properties is given by

$$
\Delta=-4 A_{1}^{3}-27 A_{2}^{2}
$$

If $\Delta>0$ there will be three distinct real roots, if $\Delta<0$ then there will be one real root (and two complex roots) and if $\Delta=0$ there can be repeated real roots. The roots of Eq. (24) are as follows:

$$
x=\left\{\begin{array}{c}
-\frac{(2 / 3)^{1 / 3} A_{1}}{\left(-9 A_{2}+\sqrt{-3 \Delta}\right)^{1 / 3}}+\frac{\left(-9 A_{2}+\sqrt{-3 \Delta}\right)^{1 / 3}}{2^{1 / 3} 3^{2 / 3}} \\
\frac{(1 \pm i \sqrt{3}) A_{1}}{2^{2 / 3} 3^{1 / 3}\left(-9 A_{2}+\sqrt{-3 \Delta}\right)^{1 / 3}}-\frac{(1 \mp i \sqrt{3})\left(-9 A_{2}+\sqrt{-3 \Delta}\right)^{1 / 3}}{2^{4 / 3} 3^{2 / 3}} .
\end{array}\right.
$$

\footnotetext{
${ }^{3}$ For bounce one must have $\alpha<0$ as shown in 35
} 
In terms of trigonometric functions the above roots can be represented as,

$$
x=\left\{\begin{array}{c}
2 \sqrt{-\frac{A_{1}}{3}} \cos \left[\frac{1}{3} \tan ^{-1}\left(\frac{\sqrt{3 \Delta}}{-9 A_{2}}\right)\right], \\
-2 \sqrt{-\frac{A_{1}}{3}} \cos \left[\frac{1}{3}\left(\pi \mp \tan ^{-1}\left(\frac{\sqrt{3 \Delta}}{-9 A_{2}}\right)\right)\right] .
\end{array}\right.
$$

In the numerical calculations we will use the above form of the roots as they are less cumbersome to handle when all the roots are real. If initially the three roots of Eq. 24 are all real then one does require a separate initial condition, specifying a particular initial root out of the three possible roots, to describe the anisotropic cosmological dynamics in $f(R)$ gravity. On the other hand if there is only one real root then the added initial condition looses its significance and the initial conditions as specified in the last section is enough to describe the cosmological dynamics.

From Eq. 19 it is seen that the anisotropy factor depends upon $a, \dot{a}, \ddot{a}$ and the integration constant $b$. More over from the form of the cubic equation followed by $x$ it can be easily seen that out of the three roots one tends to vanishes when $b \rightarrow 0$, where as the other two roots in general do not tend to zero when $b$ becomes arbitrarily small. If all the roots are real then the root which vanishes when $b$ vanishes plays an important role as in this case one can tune the value of the initial anisotropy by tuning the value of $b$. When the system admits only one real root then this root always tends to zero when $b$ tends to zero. In GR, when one deals with anisotropic Bianchi Type-I cosmology, the equation followed by the anisotropy factor is $x(t)=b / a^{3}(t)$ and hence no such complications arise.

\subsection{Starobinsky inflation}

We can now apply our formalism to get the first nontrivial result related to anisotropic cosmological dynamics in Starobinsky's model of inflation. In this model of inflation the universe inflates in absence of any hydrodynamic fluid. In quadratic gravity inflation the parameter $\alpha$ appearing in Eq. (23) is always positive which makes $f^{\prime} \equiv d f / d R>1$. In presence of anisotropy the inflating spacetime shows very fast growth in the average scale-factor $a(t)$ while the average Hubble parameter satisfies the condition $\dot{H}=-\epsilon H^{2}$ where $\epsilon$ is a slow-roll parameter. During inflation $\epsilon \ll 1$ and this condition prevails until $\epsilon \sim 1$ at the end of inflation 36]. In the excellent review on Starobinsky inflation given in Ref. [36] the authors use the slow-roll approximation to derive the properties of the inflating FLRW spacetime. In this paper we will use the conventions of 
the above reference but will not exactly apply slow-roll mechanism. We will use the full $f(R)$ theory equations as discussed in the last section with specific inflationary initial condition which gives rise to a rapidly expanding universe. In a later publication we want to generalize slow-roll conditions in quadratic gravity inflation in anisotropic Bianchi-I spacetimes.

In this subsection we show that any kind of anisotropy, if present initially, will be damped during the inflationary phase in quadratic gravity. We analytically prove our result for large initial anisotropy, the proof remains the same for small initial anisotropy. If initially the anisotropy factor was large then inflation will successfully isotropize the universe and there will be no remaining anisotropy at the end of inflation. Although this fact is known to be true in inflationary models based on GR [14], in this article we show that similar outcome is also expected in quadratic theory of inflation. First we show that the maximum anisotropy allowed in quadratic gravity, during inflation, has a maximum bound:

$$
x \leq \sqrt{6} H
$$

consequently the maximum anisotropy which can be isotropized is related with the Hubble parameter. To prove the above assumption one must note that in quadratic gravity inflation, $\rho=0$, and the inflationary phase is initiated by curvature energy density $\rho_{\text {curv }}$. The anisotropy energy contribution, in Eq. (13), is non-negative and consequently for inflation to start initially (when ideally the anisotropy effect is maximum) $\rho_{\text {curv }}>0$. The fact that the curvature energydensity appearing in the constraint Eq. (13) as:

$$
H^{2}=\frac{\kappa \rho_{\text {curv }}}{3 f^{\prime}(R)}+\frac{x^{2}}{6},
$$

cannot be negative during inflation justifies Eq. 30.

Even if the initial anisotropy present in the universe is given by the maximum bound of $x$ in Eq. (30) the anisotropy factor rapidly fades away during quadratic inflation. To show this we first note that in Starobinsky inflation $A_{1}>0$, as $|\dot{H}| \ll H^{2}$. As a result the discriminant $\Delta<0$ implying that there is only one unique real root of Eq. 24). This root is given by the top right hand side term in Eq. (28). From the expression of $A_{2}$ one can see that it rapidly diminishes in an inflating universe and one can assume $A_{1}^{3}>A_{2}^{2}$ after some time from the onset of inflation. Assuming that $A_{2} \rightarrow 0$ rapidly after inflation starts one can easily show that the relevant real root of Eq. (30) tends to zero during inflation. 


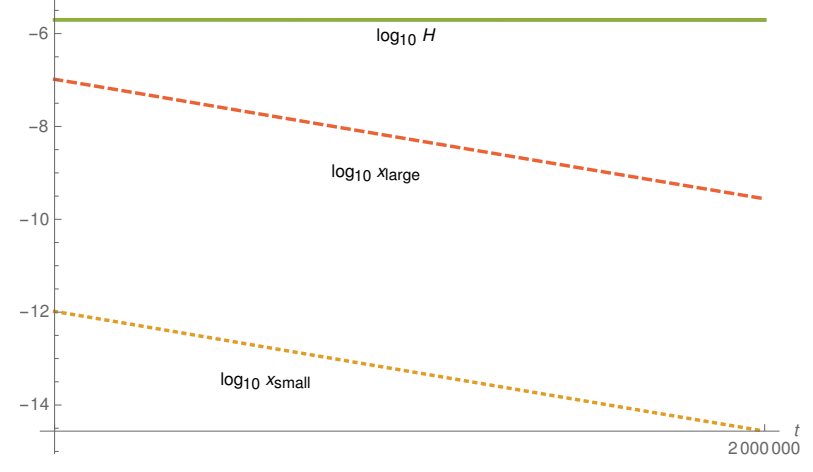

Figure 1: Plot showing evolution of $H$ and $x$ in logarithmic scale during inflationary phase. Relatively large initial anisotropy $x_{\text {large }}$ corresponding to $b=10^{-5}$ is shown by the dashed line. Small initial anisotropy $x_{\text {small }}$ corresponding to $b=10^{-10}$ is plotted by the dotted line. The Hubble parameter remains approximately the same in both the cases. Time axis spans from $10^{6}$ to $2 \times 10^{6}$ and initial $H \sim 2 \times 10^{-6}$ (for both the cases of large and small initial anisotropy). Planck units are used for time and $H$.

We will now present a numerical solution of the cosmological dynamics during inflation and point out that the anisotropy factor $x^{2} / H^{2}$ always remains sufficiently smaller and at no stages of quadratic inflation $x^{2} \approx H^{2}$. Here we assume that inflation starts at, $t_{i}=10^{6}$, in Planck units. In this unit system the actual value of a quantity is obtained by multiplying the value of the physical quantity by a particular power of Plank mass $M_{P}$. The specific power corresponds to the mass dimension of the physical quantity. In the present case the actual value of $t_{i}$ is $t_{i} M_{P}^{-1}$. In this article we will assume $M_{P} \approx 10^{19} \mathrm{GeV}$ and consequently $t_{i}=10^{-13} \mathrm{GeV}^{-1}$ expressed in energy units. Expressed in the seconds $t_{i} \approx 10^{-37} \mathrm{~s}$ and that is $10^{6}$ times Planck time expressed in seconds. The value of $\alpha$ is chosen as $\alpha=10^{12}$ which in natural units will be $10^{12} M_{P}^{-2}$. Phenomenologically one expects quadratic correction to Einstein gravity at a very early phase of the universe when $H \sim 10^{12-13} \mathrm{GeV}$ or more. For this benchmark value of the $H$ the Ricci scalar $R \sim 10^{26} \mathrm{GeV}^{2}$ assuming $x^{2}<H^{2}$. If the quadratic correction $\alpha R^{2}$ becomes effective at such a value of $R$ then $\alpha \sim 1 / R$ yielding $\alpha \sim 10^{-26} \mathrm{GeV}^{-2}=10^{12} M_{P}^{-2}$, justifying our choice of $\alpha$. The initial Hubble parameter is chosen to be $H\left(t_{i}\right)=2 \times 10^{-6}$ in Planck units, its value in standard units is $10^{13} \mathrm{GeV}$. Inflation ends at $t_{f} \approx t_{i}+70 \times H\left(t_{i}\right)^{-1}=36 \times 10^{6}$, 


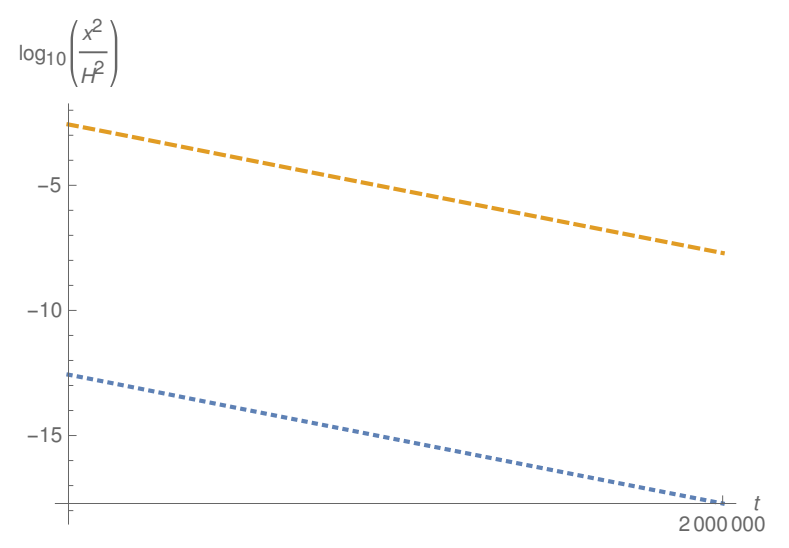

Figure 2: Evolution of the $x^{2} / H^{2}$ in logarithmic scale for the cases corresponding to relatively large anisotropy where $b=10^{-5}$, in dashed curve and relatively small anisotropy, where $b=10^{-10}$, in dotted curve. Time span and initial Hubble parameter value remains the same as specified in the caption of Fig. 1.

which corresponds to slightly more than 70 e-folds. The consistent inflationary initial conditions are written in terms of the initial values of the slow-roll parameters. The initial slow-roll parameters are chosen as $\epsilon\left(t_{i}\right)=-\dot{H} / H^{2} \approx 7 \times 10^{-3}$ and $\eta\left(t_{i}\right)=\ddot{H} /(H \dot{H}) \approx 0$. The other initial conditions are as:

$$
\begin{aligned}
a\left(t_{i}\right) & =1, \\
\dot{a}\left(t_{i}\right) & =H\left(t_{i}\right) a\left(t_{i}\right), \\
\ddot{a}\left(t_{i}\right) & =\frac{(1-\epsilon) \dot{a}\left(t_{i}\right)^{2}}{a\left(t_{i}\right)}, \\
\dddot{a}\left(t_{i}\right) & =\frac{(1-\eta \epsilon-3 \epsilon) \dot{a}\left(t_{i}\right)^{3}}{a\left(t_{i}\right)^{2}} .
\end{aligned}
$$

Although the initial conditions for inflation in the present section are written in terms of the slow-roll parameters $\epsilon$ and $\eta$ at initial time we do not evolve $\epsilon$ or $\eta$ with time or use the slow-roll parameters in any other place in our calculation. The calculation do not use slow-roll approximation and the results we present in this subsection are exact results.

To get a numerical solution, we plug the solution $x(a, \dot{a}, \ddot{a})$ into the dynamical equation, Eq. (16). The resulting dynamical equation for $a(t)$ is a fourth order ordinary differential equation for the case of quadratic $f(R)$ gravity. Looking at

\footnotetext{
${ }^{4}$ Here we apply the initial conditions for inflation on the average scale-factor and Hubble parameter and its derivatives. By consistent initial conditions we mean that these initial conditions satisfy all the constraints of quadratic gravity inflation as discussed in Ref. [36.
} 
the structure of the roots of the cubic equation, presented in the initial part of the present section, it is seen that in the present case the coefficient $A_{1}>0$ and consequently there will be only one real root of the anisotropy factor $x(t)$. This root continuously tends to zero as $b$ tends to zero.

We have plotted the numerical results showing the growth of the Hubble parameter and $x$, for small and large relative initial anisotropy, in logarithmic scales in Fig. 1. Small anisotropy, $x_{\text {small }}$, corresponds to $b=10^{-10}$ and relatively large anisotropy, $x_{\text {large }}$, corresponds to $b=10^{-5}$. For both small and large anisotropies the scale-factor and the Hubble parameter are approximately the same showing that the overall inflating nature of the system does not depend upon the initial anisotropy present in the system. The inflationary nature of the present system is shown by the near constant value of $H$ in Fig. 1. Fig. 2 shows the growth of the anisotropy factor $x^{2} / H^{2}$ in the two cases corresponding to the two $b$ values as discussed above. This plot clearly shows that anisotropy rapidly dies in quadratic gravity inflation. We have numerically verified that anisotropy gets wiped out after the first two or three e-folds of inflation and consequently suppression of anisotropy happens very efficiently in quadratic inflation. Here it must be noted that we cannot arbitrarily increase $b$ as when $b>10^{-4}$ the consistency condition in Eq. (30) is violated and the system does not inflate any more. Our formalism shows both analytically and numerically that Starobinsky inflation is safe from initial anisotropy.

\subsection{Contraction in toy model of quadratic bounce}

In this subsection we discuss anisotropic contraction phase in a simple and partly unstable model, guided by quadratic gravity. The presentation in this subsection is more like a toy model analysis which shows the complexities of anisotropic contraction in polynomial $f(R)$ gravity models. In general the solution of Eq. (18) becomes a polynomial equation in $x$ and for higher polynomial orders (compared to quadratic order) the algebraic equations do not yield analytic solutions. The quadratic gravity bounce model, where $\alpha<0$ is the simplest polynomial bounce model, where the intricacies of anisotropy generation during the contraction phase can be semi-analytically shown.

The issue of anisotropy generation during a contraction phase is very important as anisotropy may get enhanced during this phase as it happens in GR based models of cosmological bounce. We want to see how anisotropy grows in Bianchi- 
I models in the contraction phase in quadratic $f(R)$ gravity. In the present case we will assume the existence of hydrodynamic matter and $\alpha<0$ as these conditions are required for a subsequent bounce [35]. Before we proceed we will like to make some remarks related to the choice of the sign of $\alpha$. The negative sign of $\alpha$ implies that $f^{\prime}(R)$ is not always positive. We can choose our dynamical system to be such that it satisfies $f^{\prime}(R)>0$ for some range of $R$, as done in the present paper. The importance of negative $\alpha$ quadratic model is that one can have a cosmological bounce in this restricted regime of $R$, where $R<1 /(2|\alpha|)$ for stability. There is another source of instability in the present case, related to the negative sign of $\alpha$. In such models $f^{\prime \prime}<0$ which may lead to instabilities first proposed by Dolgov and Kawasaki [37] and later by V. Faraoni [38]. In the present model one cannot get rid of Dolgov and Kawasaki instability 5 , consequently in light of the stability issues we will like to interpret the present model of bounce as a toy model whose sole purpose is to describe the nonlinear growth of anisotropy. In the a later section we will apply or formalism in a stable gravitational model.

In GR it is known that anisotropy suppression during contraction phase requires the presence of an ultra-stiff matter component with $\omega(=P / \rho)>1$. The presence of an ultra-stiff matter component can produce a slow contraction phase where preexisting anisotropy is suppressed 6 . In the present case we will see that a power law contraction phase may suppress initial anisotropy in quadratic $f(R)$ cosmology.

We assume that during the contracting phase $t<0$ and bounce occurs at $t=0$. During the contracting phase the scale-factor decreases as

$$
a(t) \propto(-t)^{n}, \text { where } 0<n<1
$$

and consequently

$$
H=\frac{n}{t} .
$$

From physical considerations one can choose $\alpha=-10^{12}$ in Planck units 35. Eliminating $\rho$ in Eq. (16) by using Eq. (13) we get,

$$
\omega=\frac{\left(4 \dot{H}+6 H^{2}+x^{2}\right) f^{\prime}+2 \kappa P_{\text {curv }}}{\left(x^{2}-6 H^{2}\right) f^{\prime}+2 \kappa \rho_{\text {curv }}} .
$$

\footnotetext{
${ }^{5}$ In Ref. 39 it was explicitly shown that polynomial $f(R)$ gravity theories which accommodate bouncing solutions cannot satisfy $f^{\prime}>0$ and $f^{\prime \prime}>0$ for all values of Ricci scalar.

${ }^{6}$ Sometimes this phase of slow contraction under the dominance of a ultra-stiff matter is called the ekpyrotic phase [10].
} 


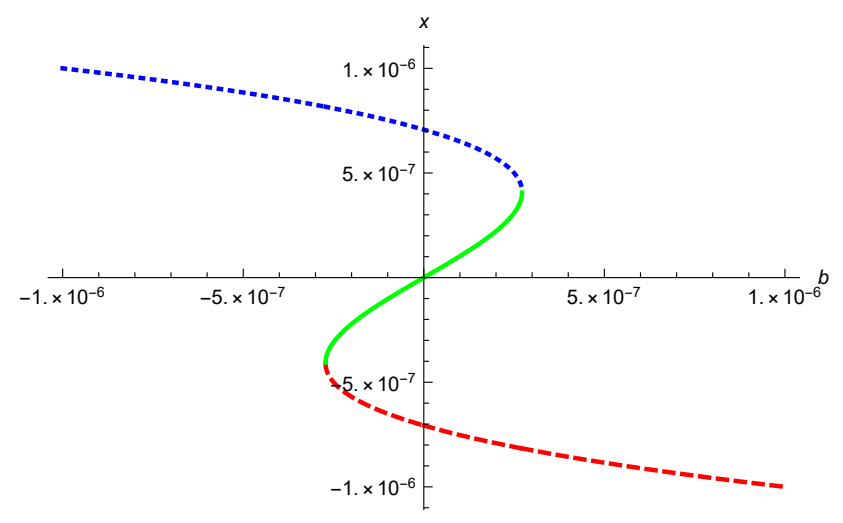

Figure 3: Plot of initial anisotropy $x$ with respect to parameter $b$ at $t=-10^{10}$. The dashed and the continuous parts correspond to the (second and third) roots with the minus/plus signs after $\pi$ in the second line on the right hand side of Eq. (29). The dotted part corresponds to the (first) root on the first line on the right hand side of Eq. (29). All the three roots are real near $b=0$.

In the present case the above equation yields the equation of state for the barotropic matter when one specifies the particular nature of the scale-factor.

Determining the form of the time evolution of anisotropy factor reduces to finding the root(s) of Eq. (19). One can have various phases of anisotropy development during a cosmological evolution depending upon the roots of Eq. (19). In this paper we will particularly focus on the contracting phase of the universe leading to a cosmic bounce. We present the results for the popular quadratic $f(R)$ model which actually accommodates a cosmological bounce [35], [40], [41], [42]. The nature of the anisotropic contraction phase predicted in this model will give a glimpse of the interesting effects of $f(R)$ models of anisotropic contraction in the Bianchi-I spacetime. The plot in Fig. 3 shows the nature of the roots at time $t=-10^{10}$ in Planck units. The time period of contraction is chosen in such a way that all the constraints as $f^{\prime}(R)>0$ and $\rho>0$ are maintained during this phase of contraction. As the power law contraction can never lead to a bounce the constraints compel us to terminate the power law contraction process some time before the bounce and in this paper we use the time interval $-10^{10} \leq t \leq-10^{7}$. The scale-factor during this time is assumed to be $a(t)=\left(-t / 10^{10}\right)^{n}$ such that $a\left(t=-10^{10}\right)=1$. The nature of the roots show that below a certain $b$ value and above a certain $b$ value there is only one real root. Near $b=0$ the system admits three real roots of $x(t)$. We have verified that the nature of the root structure, as 


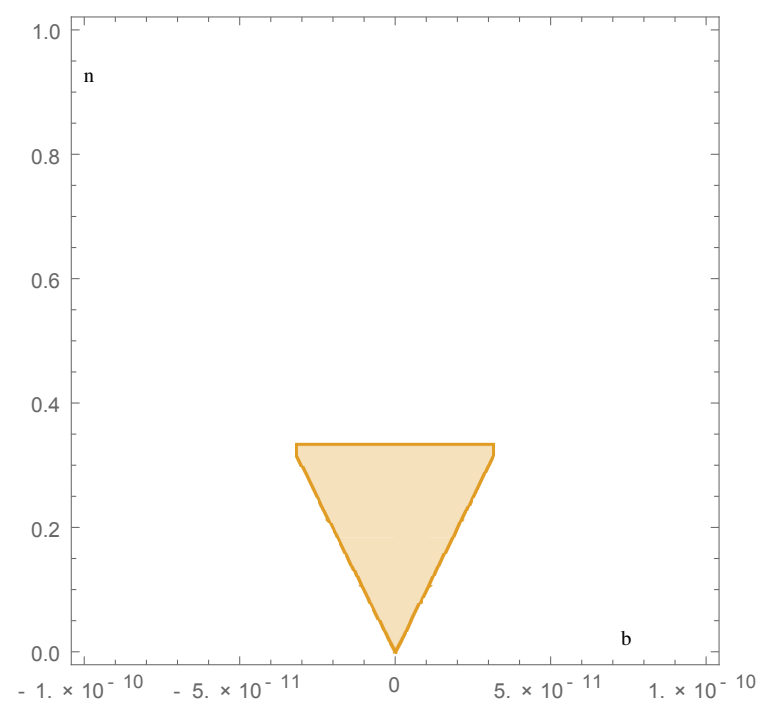

Figure 4: Region in the $b-n$ plane giving rise to decreasing $x^{2} / H^{2}$. Here the abscissa is specified by the $b$ values and the ordinate is specified by $n$ values.

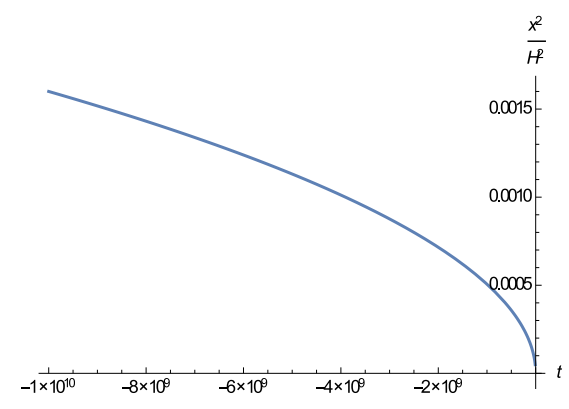

Figure 5: Decrease of anisotropy in time when $b, n$ lies in the shaded region in Fig. 4. The specific $b, n$ value chosen for the plot is given in the text.

specified in Fig. 3, does feebly depend upon $n$ in the interval $0 \leq n \leq 1$. The plot in Fig. 3 shows three branches in three colors. The middle green (continuous line) branch smoothly matches to the blue (dotted) branch above and the red (dashed) one below. The continuous branch specifies a root of Eq. (24) which is real near $b=0$ and gives rise to small values of anisotropy factor $x_{0}=x\left(t=-10^{10}\right)$ initially. The dashed and dotted branches specify the other roots which are large for regions near $b=0$. The connection of the three regions in the figure with the roots in Eq. 29) are specified in the caption of Fig. 3. As time evolves the nature of the plot in Fig. 3 changes but the general structure of the plot always remains qualitatively similar as the one plotted at the initial time.

The dynamics of anisotropy growth depends upon the parameters $b$ and $n$. We can specify the region in the $b-n$ plane which gives rise to decreasing anisotropy. The plot in Fig. 4 shows such a region in the $b-n$ plane. The plot is done at $t=-10^{10}$, the initial time, when the region is most constrained. In Fig. 5 we show how $x^{2} / H^{2}$ varies in time if one uses any value of $b, n$ in the shaded region in Fig.4. In particular we have chosen $b=10^{-12}$ and $n=1 / 4$. The above information shows that for some parameter values a power law contraction can indeed suppress small anisotropy in quadratic gravity. Physically anisotropy 
suppression for some regions in the $b-n$ plane in the contracting phase is not surprising as both $x$ and $H$ do increase in time during contraction when $b$ and $n$ belongs to the shaded region in Fig. 4 (as expected) but $H$ increases more than $x$ in time and as a consequence $x^{2} / H^{2}$ decreases with time. For some parameter space $H$ can grow faster than $x$ in time, when anisotropy factor decreases, and for other parameter values $x$ increases more than $H$ in time making the contracting universe completely anisotropic.

\section{Anisotropy growth in exponential gravity}

Recently it has been shown [43] that one can get bouncing solutions and expanding universe solutions in a unstable de-Sitter point in exponential gravity where

$$
f(R)=\frac{1}{\alpha} e^{\alpha R}, \quad \alpha>0
$$

where $\alpha$ is a dimensional, real constant. In the present case as $\alpha>0$ we have $f^{\prime}(R)>0$ and $f^{\prime \prime}(R)>0$ and the theory remains stable for all values of $R$. It was shown in Ref. [43] that exponential gravity do admit some exact solutions. One exact solution is a bouncing solution and another one is an expanding universe solution with constant Hubble parameter which takes place at a de-Sitter point. As we know two exact solutions in exponential gravity we can investigate about the growth of anisotropy in these two cases.

In the present case the solution of anisotropy factor is,

$$
x=\frac{b}{f^{\prime}(R)}=\frac{b}{a^{3} e^{\alpha R}}=\frac{b}{a^{3} e^{6 \alpha\left(\dot{H}+2 H^{2}\right)}} e^{-\alpha x^{2}} .
$$

This is a transcendental equation in $x(t)$. The form of the above equation also shows that there will be only one real solution at any given time, given graphically by the intersection of a straight line $y=x$ and a Gaussian $y=\frac{b}{a^{3} e^{6 \alpha\left(\dot{H}+2 H^{2}\right)}} e^{-\alpha x^{2}}$. The small anisotropy solution can be obtained analytically. If we want to see how small anisotropy, defined by all the $x$ values which satisfy $x^{2} \ll H^{2}$, develop we may approximate the last equation as:

$$
x \sim \frac{b}{a^{3} e^{\alpha R_{\text {iso }}}} .
$$

where $R_{\text {iso }} \equiv 6\left(\dot{H}+2 H^{2}\right)$ and $a$ is the average scale-factor. We discuss the evolution of small anisotropy for the two exact solutions of exponential gravity which was extensively discussed in 43 . 


\subsection{Bouncing solution}

Exponential gravity has an exact bouncing solution, where the scale-factor is given by $a(t)=e^{A t^{2}}$ where $A$ is a real constant. Bounce happens in the presence of matter at $t=0$, and the conditions for an exact solution requires $\alpha A=1 / 48$ and the equation of state of matter $\omega=-4 / 3$. In the present case $R_{\text {iso }}=$ $12 A\left(1+4 A t^{2}\right)$ and consequently for small anisotropy we must have

$$
x(t)=\frac{b}{e^{1 / 4}} e^{-4 A t^{2}}
$$

which shows that how the anisotropy factor changes with time. The real indicator of anisotropy is the ratio $x^{2} / H^{2}$ and in our present case

$$
\frac{x^{2}}{H^{2}}=\frac{b^{2}}{4 A^{2} \sqrt{e}} \frac{e^{-8 A t^{2}}}{t^{2}} .
$$

A small anisotropy ratio at $t \rightarrow-\infty$ remains smaller than one for some time but then after some finite time $x^{2} \sim H^{2}$ and cosmic dynamics is guided by the anisotropy factor leading to an instability. From our simple analysis we see that the specific bouncing scenario presented in this section is unstable under small values of anisotropy.

\subsection{Expansion with constant Hubble parameter at the de- Sitter point}

Exponential gravity has another exact, constant Hubble parameter solution at a de-Sitter point where $R_{\text {iso }}=2 / \alpha$. The scale-factor of the universe at the de-Sitter point is $a(t)=e^{H t}$ and this is a vacuum solution when $H^{2}=1 /(6 \alpha)$ is satisfied. In the present case small anisotropy grows as

$$
x(t)=\frac{b}{e^{2}} e^{-3 H t} .
$$

and consequently

$$
\frac{x^{2}}{H^{2}}=\frac{6 \alpha b^{2}}{e^{4}} e^{-\sqrt{(6 / \alpha)} t} .
$$

In this case we see that small anisotropy decreases with time. This analysis is not complete as we do not know how large anisotropy behaves in these situations. To tackle the question of large anisotropy one has to purely rely on numerical methods. 


\section{Conclusion}

This paper presents the general results for anisotropic cosmological development in Bianchi-I model in metric $f(R)$ gravity. The initial part of the paper develops the formalism which can be used to track cosmological development in homogeneous and anisotropic Bianchi-I model. The formalism developed is dynamically complete and can predict the development of all the relevant cosmological and fluid parameters in cosmological time. The methods developed in this paper can be applied to expanding as well as contracting phase of the universe. As anisotropy reduces in the expanding phase in GR it does not mean that this rule will be generally followed in $f(R)$ cosmology as the equation predicting anisotropy growth is non-linear in nature and may have surprises in store. Our preliminary calculations predicts that inflation in quadratic $f(R)$ cosmology, in Bianchi-I spacetime, indeed suppresses anisotropy. The results related to inflationary models in anisotropic spacetimes in $f(R)$ theory are presented in full details in the present paper. We first show that for Bianchi-I type models one can analytically prove that anisotropy fades away in quadratic gravity inflation. We numerically show the validity of our analytic proof.

As anisotropy development demands special attention in the contracting phase in cosmological models based on GR our aim was to see how the problem translates into $f(R)$ cosmology. In this article we tried to verify whether anisotropy subsides in the $f(R)$ theory driven contraction phase. The result we obtain is complex and opens up new areas of research. We have chosen quadratic $f(R)$ theory to illustrate our results as in this case most of the calculations can be done analytically although the bouncing scenario is gravitationally unstable. For any other higher order polynomial $f(R)$ one has to use numerical methods to determine the solutions of the differential equation predicting anisotropy dynamics. Our work shows the qualitative nature of the cosmological system, guided by quadratic gravity, undergoing anisotropic contraction and we expect qualitatively similar but quantitatively much more formidable results for other complicated, gravitationally stable polynomial $f(R)$ cosmologies. Even in the case of quadratic gravity the various results coming out from our formalism is non-trivial. We have pointed out that even when we restrict the cosmological dynamics by enforcing conditions as $f^{\prime}>0$ and $\rho>0$ there appears various regions in the $n, b$ plane which gives rise to different kind of anisotropy growth. For some possible cosmological evolutions we have shown that anisotropy reduces with time. There exists 
other possibilities where anisotropy increases with time during the contraction phase in quadratic gravity.

As quadratic $f(R)$ theory cosmological bounce is more like a toy model because in this case the cosmological dynamics is unstable we have tried to show the applicability of our result in stable exponential gravity model which admits an exact bouncing solution. In this case we have not presented a general result but has focussed on small anisotropy growth. Our result shows that the exact bouncing solution in exponential gravity model is unstable and consequently the cosmological system will tend towards an instability in the contraction phase. We have also showed that small anisotropy subsides in the expansion phase at the de-Sitter point in exponential gravity.

The present paper shows that the issue about anisotropy in Bianchi-I spacetimes in metric $f(R)$ gravity is a nonlinear problem which may lead to very complex conditions in contracting regions of a bouncing model. For polynomial gravity theories the cosmological contraction process is much involved and requires full numerical simulation to find out meaningful results. For expanding cosmologies our theory has given expected results, the amount of anisotropy goes down with expansion. But whether anisotropy will reduce for all kinds of expansion processes requires a more general proof and we hope we will able to show more general and formal work in these lines in the near future.

\section{References}

[1] J. Wainwright, A. A. Coley, G. F. R. Ellis and M. Hancock, Class. Quant. Grav. 15, 331 (1998). doi:10.1088/0264-9381/15/2/008

[2] C. M. Chen and W. F. Kao, Phys. Rev. D 64, 124019 (2001) doi:10.1103/PhysRevD.64.124019 hep-th/0104101.

[3] C. M. Chen and W. F. Kao, hep-th/0201188.

[4] J. Barrow and H. Kodama, Class. Quant. Grav. 18, 1753 (2001) doi:10.1088/0264-9381/18/9/310 gr-qc/0012075.

[5] T. Pereira and C. Pitrou, Comptes Rendus Physique 16, 1027 (2015) doi:10.1016/j.crhy.2015.09.002 arXiv:1509.09166 [astro-ph.CO]]. 
[6] P. Anninos, R. A. Matzner, T. Rothman and M. P. Ryan, Phys. Rev. D 43, 3821 (1991). doi:10.1103/PhysRevD.43.3821

[7] Y. Kitada and K. i. Maeda, Class. Quant. Grav. 10, 703 (1993). doi:10.1088/0264-9381/10/4/008

[8] Y. Kitada and K. i. Maeda, UTAP-149-92, WU-AP-24-92, C92-08-19.

[9] T. Q. Do and W. F. Kao, Phys. Rev. D 96, no. 2, 023529 (2017). doi:10.1103/PhysRevD.96.023529

[10] D. Garfinkle, W. C. Lim, F. Pretorius and P. J. Steinhardt, Phys. Rev. D 78, 083537 (2008) doi:10.1103/PhysRevD.78.083537 arXiv:0808.0542 [hepth]].

[11] V. Bozza and M. Bruni, JCAP 0910, 014 (2009) doi:10.1088/14757516/2009/10/014 arXiv:0909.5611 [hep-th]].

[12] J. D. Barrow and K. Yamamoto, Phys. Rev. D 82, 063516 (2010) doi:10.1103/PhysRevD.62.063516 arXiv:1004.4767 [gr-qc]].

[13] J. D. Barrow and C. Ganguly, Class. Quant. Grav. 33, no. 12, 125004 (2016) doi:10.1088/0264-9381/33/12/125004 arXiv:1510.01095 [gr-qc]].

[14] R. M. Wald, Phys. Rev. D 28, 2118 (1983). doi:10.1103/PhysRevD.28.2118

[15] V. A. Belinsky, I. M. Khalatnikov and E. M. Lifshitz, Adv. Phys. 19, 525 (1970). doi:10.1080/00018737000101171

[16] M. Sharma, arXiv:1501.01958 [gr-qc].

[17] S. Panda and M. Sharma, Astrophys. Space Sci. 361, no. 2, 87 (2016) doi:10.1007/s10509-015-2594-y arXiv:1501.01765 [gr-qc]].

[18] D. M. Solomons, P. Dunsby and G. Ellis, Class. Quant. Grav. 23, 6585 (2006) doi:10.1088/0264-9381/23/23/001 [gr-qc/0103087].

[19] Y. F. Cai, E. McDonough, F. Duplessis and R. H. Brandenberger, JCAP 1310, 024 (2013) doi:10.1088/1475-7516/2013/10/024 arXiv:1305.5259 [hep-th]]. 
[20] Y. F. Cai, Sci. China Phys. Mech. Astron. 57, 1414 (2014) doi:10.1007/s11433-014-5512-3 arXiv:1405.1369 [hep-th]].

[21] S. Nojiri, S. D. Odintsov and V. K. Oikonomou, Phys. Rept. 692, 1 (2017) doi:10.1016/j.physrep.2017.06.001 [arXiv:1705.11098 [gr-qc]].

[22] D. Muller, A. Ricciardone, A. A. Starobinsky and A. Toporensky, arXiv: 1710.08753 [gr-qc].

[23] A. Toporensky and D. Muller, Gen. Rel. Grav. 49, no. 1, 8 (2017) doi:10.1007/s10714-016-2172-9 arXiv:1603.02851 [gr-qc]].

[24] K. i. Maeda, Phys. Rev. D 37, 858 (1988). doi:10.1103/PhysRevD.37.858

[25] S. Cotsakis and G. Flessas, Phys. Lett. B 319, 69 (1993). doi:10.1016/03702693(93)90783-E

[26] H. J. Schmidt, Class. Quant. Grav. 5, 233 (1988). doi:10.1088/0264$9381 / 5 / 1 / 027$

[27] A. A. Starobinsky, Phys. Lett. 91B, 99 (1980). doi:10.1016/03702693(80)90670-X

[28] G. Leon, Int. J. Mod. Phys. E 20, 19 (2011) doi:10.1142/S0218301311040037 [arXiv:1403.1984 [gr-qc]].

[29] G. Leon and E. N. Saridakis, Class. Quant. Grav. 28, 065008 (2011) doi:10.1088/0264-9381/28/6/065008 arXiv:1007.3956 [gr-qc]].

[30] A. De Felice, A. E. Gumrukcuoglu, , C. Lin and S. Mukohyama, JCAP 1305, 035 (2013) doi:10.1088/1475-7516/2013/05/035 arXiv:1303.4154 [hep-th]].

[31] J. Middleton, Class. Quant. Grav. 27, 225013 (2010) doi:10.1088/02649381/27/22/225013 arXiv:1007.4669 [gr-qc]].

[32] M. F. Shamir, J. Exp. Theor. Phys. 123, no. 6, 979 (2016). doi:10.1134/S1063776116150152

[33] T. Clifton and J. D. Barrow, Class. Quant. Grav. 23, 2951 (2006) doi:10.1088/0264-9381/23/9/011 gr-qc/0601118. 
[34] D. K. Banik, S. K. Banik and K. Bhuyan, Indian J. Phys. 91, no. 1, 109 (2017). doi:10.1007/s12648-016-0898-6

[35] N. Paul, S. N. Chakrabarty and K. Bhattacharya, JCAP 1410, no. 10, 009 (2014) doi:10.1088/1475-7516/2014/10/009 [arXiv:1405.0139 [gr-qc]].

[36] A. De Felice and S. Tsujikawa, Living Rev. Rel. 13, 3 (2010) doi:10.12942/lrr-2010-3 [arXiv:1002.4928 [gr-qc]].

[37] A. D. Dolgov and M. Kawasaki, Phys. Lett. B 573, 1 (2003) doi:10.1016/j.physletb.2003.08.039 astro-ph/0307285].

$\begin{array}{lllllll} & \text { [38] V. Faraoni, } & \text { Phys. Rev. D } & \text { 74, } & 104017 & \text { (2006) }\end{array}$ doi:10.1103/PhysRevD.74.104017 astro-ph/0610734.

[39] K. Bhattacharya and S. Chakrabarty, JCAP 1602, no. 02, 030 (2016) doi:10.1088/1475-7516/2016/02/030 [arXiv:1509.01835 [gr-qc]].

[40] S. Carloni, P. K. S. Dunsby and D. M. Solomons, Class. Quant. Grav. 23, 1913 (2006) doi:10.1088/0264-9381/23/6/006 [gr-qc/0510130].

[41] K. Bamba, A. N. Makarenko, A. N. Myagky, S. Nojiri and S. D. Odintsov, JCAP 1401, $008 \quad(2014) \quad$ doi:10.1088/1475-7516/2014/01/008 arXiv:1309.3748 [hep-th]].

[42] C. Barragan and G. J. Olmo, Phys. Rev. D 82, 084015 (2010) doi:10.1103/PhysRevD.82.084015 arXiv:1005.4136 [gr-qc]].

[43] P. Bari, K. Bhattacharya and S. Chakraborty, Universe 4, no. 10, 105 (2018). doi:10.3390/universe4100105 